\title{
Abortion care-seeking and reproductive rights violation in health facilities: evidence from six states of India
}

\author{
Manas Ranjan Pradhan ${ }^{1 *}$, Chander Shekhar ${ }^{1}$, Manoj Alagarajan ${ }^{1}$, Harihar Sahoo ${ }^{2}$
}

\begin{abstract}
${ }^{1}$ Department of Fertility and Social Demography, ${ }^{2}$ Department of Family and Generation, International Institute for Population Sciences, Mumbai, Maharashtra, India
\end{abstract}

Received: 23 November 2021

Accepted: 14 December 2021

\section{*Correspondence:}

Dr. Manas Ranjan Pradhan,

E-mail: manasiips@gmail.com

Copyright: (C) the author(s), publisher and licensee Medip Academy. This is an open-access article distributed under the terms of the Creative Commons Attribution Non-Commercial License, which permits unrestricted non-commercial use, distribution, and reproduction in any medium, provided the original work is properly cited.

\begin{abstract}
Background: Unsafe abortion continues to draw the researcher's attention due to its close association with maternal morbidity and mortality. Empirical evidence on the role of health facilities in providing safe abortion care addressing the reproductive rights of Indian women is limited.

Methods: Using data from the 2015 'unintended pregnancy and abortion in India' study, the present paper aimed to understand the violation of the reproductive rights of abortion care seekers in health facilities $(n=4001)$ in six states of India. The health facilities were sampled using a stratified random sampling strategy. Univariate and bivariate analysis was carried out using SPSS (V 25) on cleaned and weighted data.

Results: A sizeable percentage of public and private health facilities across states found seeking the consent of the husband or family members before abortion provision, compel women to adopt contraception and turn away abortion seekers, commonly citing non-medical reasons. The provision of post-abortion complications services is usually not $24 / 7$, even at the primary health centre level, hindering access to an urgent health care need.

Conclusions: There is a need to improve access to facility-based abortion services, especially in underserved rural areas, by ensuring that all public-sector facilities have adequate equipment and supplies, including MMA drugs and trained providers. Sensitization of health care providers about the importance of ethical issues and women's reproductive rights is urgently required to ensure safe, legal, and accessible abortion care.
\end{abstract}

Keywords: Abortion care, Health facilities, India, Reproductive rights

\section{INTRODUCTION}

The right to legal and safe abortion has been recognized and ratified as a women's reproductive right, which needs emphasis from a broader perspective of individual freedom and human right. ${ }^{1,2}$ Unsafe abortion continues to draw the researcher's attention due to its close association with maternal morbidity and mortality. Of 56 million induced abortions that occurred worldwide during 2012$14,45 \%$ were unsafe, and $97 \%$ of these unsafe abortions occurred in developing countries. ${ }^{3}$ The abortion rate declined markedly in developed regions, from 46 to 27 per 1,000 , but remained roughly the same (36 per 1000) in developing regions between 1990-1994 and 2010-2014. ${ }^{4}$ In India, $15 \cdot 6$ million abortions took place in 2015 , giving an abortion rate of 47 per 1000 women aged $15-49$ years. ${ }^{5}$ About 3.4 million abortions (22\%) of those 15.6 million were provided in health facilities.

Many women in India still lack access to safe abortion care, despite legalizing abortion through the MTP act of 1971. Weak regulation of public and private sector services, a physician-only policy that excludes mid-level providers, and low registration of rural compared to urban clinics are the barriers. ${ }^{6}$ Stillman et al further highlighted limited access to public and private sector facilities, financial barriers to safe services, regulatory factors affecting access to medical abortion, providers' knowledge and attitudes, lack of awareness of abortion laws, and stigma surrounding abortion affects women's 
abortion-seeking behavior. ${ }^{7}$ Unsafe abortion contributes to morbidity and mortality in India, and the morbidity from unsafe abortion is considered a severe problem. ${ }^{8,9}$ Unsafe abortions are strongly associated with maternal morbidity from complications such as hemorrhage, sepsis, peritonitis, and trauma to the cervix, vagina, uterus, and abdominal organs. ${ }^{10}$ Banerjee revealed that 12,000 deaths each year result from abortion-related complications in India. ${ }^{11}$ The literature further suggests that women's reproductive rights are considered a collective decision of the Indian family, not the individual women it affects. ${ }^{12}$

A significant proportion of Indian women obtain illegal and potentially unsafe abortions, often exposing themselves to adverse health outcomes, including death. Empirical studies on the role of health facilities in providing safe abortion care ensuring women's reproductive rights are rare. The present study aims to shed light on the violation of the reproductive rights of women seeking abortion services in health facilities across six states. Specifically, the study seeks to understand the responsiveness of the health facilities/providers and identify the opportunities (stage of abortionseeking/location of facility/ownership of facility/areas to focus) for intervention at the health facilities to address women's reproductive rights. The findings are useful for policy and programs aimed at enhanced access to safe abortion care under the broader framework of women's reproductive rights.

\section{METHODS}

This paper used data from the 'unintended pregnancy, and abortion in India (UPAI)' study carried out by the International Institute for Population Sciences (IIPS), Mumbai, Population Council, New Delhi, and Guttmacher Institute, New York, in 2015. The UPAI study aimed to provide quality data on the availability and use of abortion care and generate new estimates of the incidence of abortion and unintended pregnancy in India.

The study was carried out in six selected states, i.e., Assam, Bihar, Gujarat, Madhya Pradesh, Tamil Nadu, and Uttar Pradesh, addressing regional representation and accounts for $45 \%$ of all women of reproductive age in India. The UPAI study comprises two surveys, i.e., health facilities survey (HFS) and health professional survey (HPS). The facilities in the HFS were sampled using a stratified random sampling strategy. Data for this analysis come from specific questions on the provision of induced abortion and post-abortion complication care (PAC) services gathered through the HFS. Data were collected using face-to-face structured interviews with senior staff who had worked in the facility for at least six months and who were identified as most knowledgeable about abortion provision at their facility. Informed consent procedures were followed, and only those respondents who voluntarily consented to participate in the survey were included. The detailed study design, data collection procedure, sampling, and sample size has been published in the Lancet Global Health. ${ }^{5}$ The present analysis is based on a sample of 4001 unweighted public and private healthcare facilities providing any abortion care across six surveyed states. Analysis has been carried out using SPSS (V 25) on cleaned and weighted data $(n=19634)$. In the study, facilities have been categorized by ownership, i.e., public and private, and by location, i.e., rural and urban.

\section{RESULTS}

\section{Inadequate provision of abortion care services}

Eighty-five percent of the surveyed health facilities in the state of Madhya Pradesh provide any abortion care. The corresponding figures are $60 \%$ in Gujarat, $56 \%$ in Bihar, $53 \%$ in Tamil Nadu, $45 \%$ in Uttar Pradesh, and only $35 \%$ in Assam (Figure 1). There is a wide variation in the provision of any abortion care by ownership of the health facilities- a higher percentage of the private health facilities provide any abortion care in all the states under study. Except for Madhya Pradesh (71\%), less than onethird of the public facilities in other states offer any abortion-related care. This includes the majority of the higher-level facilities- hospitals and community health centers (CHCs). Less than a quarter of the primary health center (PHCs) provide any abortion-related care in the surveyed states, except Madhya Pradesh, where half of the PHCs are found to provide abortion-related care.

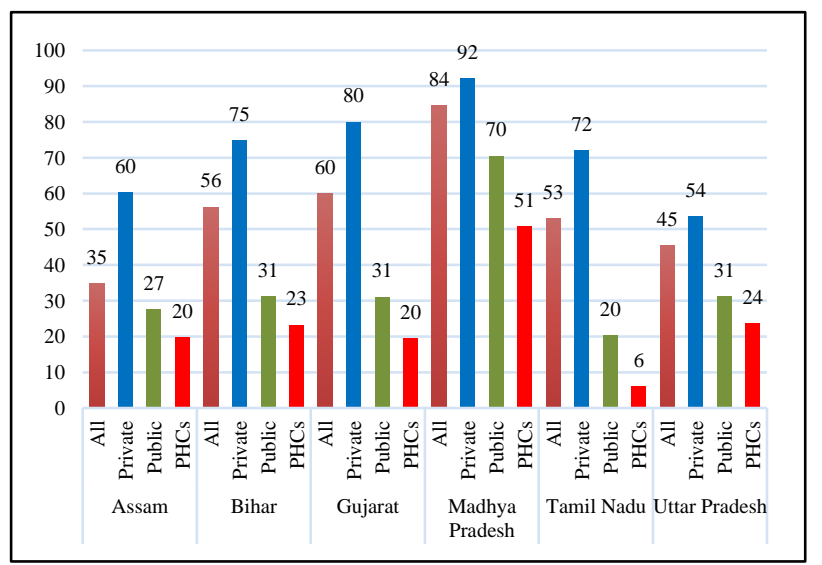

Figure 1: Percentage of health facilities providing any abortion care by ownership and type of facility, 2015.

Provision of both induced abortion and PAC services among the health facilities providing any abortion-related care varies from $38 \%$ in Uttar Pradesh to $71 \%$ in Tamil Nadu (Table 1). Irrespective of the state, a lower percentage of public facilities than those privately owned facilities provide both induced abortion and PAC services. For example, in Bihar, only $31 \%$ of the public facilities offer both induced abortion and PAC services compared with $67 \%$ of the private facilities. Nearly three-fifths (59\%) of the facilities providing abortion care in Uttar Pradesh only provide PAC. The corresponding figure is 39\% in Gujarat, 34\% each in Madhya Pradesh and Bihar, $30 \%$ in Assam, and 11\% in Tamil Nadu. Eighteen percent 
of the facilities providing any abortion care in Tamil Nadu give only induced abortion compared with three percent of the facilities in Uttar Pradesh. A higher percentage of the facilities providing any abortion care are located in urban areas in all six states. In Tamil Nadu, almost all (95\%) of these facilities are found in urban areas.

Table 1: Among facilities offering any abortion-related care, number and percentage distributions of facilities offering induced abortion, post-abortion care or both, by ownership and location, by state, 2015.

\begin{tabular}{|c|c|c|c|c|c|c|c|}
\hline \multirow[b]{2}{*}{ States } & \multirow{2}{*}{\multicolumn{2}{|c|}{$\begin{array}{l}\text { Number (weighted) of } \\
\text { facilities offering any } \\
\text { abortion-related services }\end{array}$}} & \multicolumn{3}{|c|}{$\%$ distribution by type of service offered } & \multicolumn{2}{|c|}{$\%$ distribution by location } \\
\hline & & & Abortion only & $\begin{array}{l}\text { Post-abortion } \\
\text { care only }\end{array}$ & Both & Urban & Rural \\
\hline \multirow{3}{*}{ Assam } & All & 588 & 3.8 & 30.3 & 65.9 & 54.9 & 45.1 \\
\hline & Public & 359 & 6.2 & 37.0 & 56.8 & 26.1 & 73.9 \\
\hline & Private & 229 & 0.0 & 19.8 & 80.2 & 100.0 & 0.0 \\
\hline \multirow{3}{*}{ Bihar } & All & 2,838 & 6.4 & 34.2 & 59.4 & 68.6 & 31.4 \\
\hline & Public & 629 & 4.6 & 63.9 & 31.4 & 40.2 & 59.8 \\
\hline & Private & 2,209 & 6.9 & 25.7 & 67.4 & 76.7 & 23.3 \\
\hline \multirow{3}{*}{ Gujarat } & All & 2,294 & 11.2 & 39.3 & 49.5 & 69.3 & 30.7 \\
\hline & Public & 484 & 7.0 & 46.9 & 46.1 & 26.6 & 73.4 \\
\hline & Private & 1,811 & 12.3 & 37.3 & 50.4 & 80.7 & 19.3 \\
\hline \multirow{3}{*}{$\begin{array}{l}\text { Madhya } \\
\text { Pradesh }\end{array}$} & All & 4,427 & 8.7 & 33.6 & 57.7 & 78.7 & 21.3 \\
\hline & Public & 1,302 & 11.5 & 48.5 & 40.0 & 55.2 & 44.8 \\
\hline & Private & 3,125 & 7.6 & 27.3 & 65.1 & 88.5 & 11.5 \\
\hline \multirow{3}{*}{$\begin{array}{l}\text { Tamil } \\
\text { Nadu }\end{array}$} & All & 3,235 & 18.1 & 10.9 & 71.0 & 95.0 & 5.0 \\
\hline & Public & 459 & 22.2 & 18.6 & 59.3 & 72.1 & 27.9 \\
\hline & Private & 2,776 & 17.5 & 9.6 & 72.9 & 98.8 & 1.2 \\
\hline \multirow{3}{*}{$\begin{array}{l}\text { Uttar } \\
\text { Pradesh }\end{array}$} & All & 6,252 & 3.1 & 58.8 & 38.1 & 61.5 & 38.5 \\
\hline & Public & 1,569 & 3.0 & 64.9 & 32.1 & 30.1 & 69.9 \\
\hline & Private & 4,682 & 3.1 & 56.8 & 40.1 & 72.0 & 28.0 \\
\hline
\end{tabular}

Table 2: Among facilities providing induced abortion services, proportion and percentage distribution offering each abortion method, by ownership and type of facility, 2015.

\begin{tabular}{|c|c|c|c|c|c|c|c|c|}
\hline \multirow{2}{*}{ Facilities } & & \multicolumn{3}{|c|}{$\%$ of facilities offering each method } & \multicolumn{3}{|c|}{$\begin{array}{l}\% \text { distribution of facilities by method } \\
\text { category }\end{array}$} & \multirow[b]{2}{*}{$\mathbf{N}$} \\
\hline & & $\begin{array}{l}\text { MMA } \\
\text { (combi } \\
\text { packs) }\end{array}$ & $\begin{array}{l}\text { Vacuum } \\
\text { aspiration }\end{array}$ & $\begin{array}{l}D \text { and } C / D \\
\text { and } E\end{array}$ & $\begin{array}{l}\text { Only } \\
\text { medical } \\
\text { abortion }\end{array}$ & $\begin{array}{l}\text { Only } \\
\text { surgical } \\
\text { abortion }\end{array}$ & $\begin{array}{l}\text { Both medical } \\
\text { and surgical } \\
\text { abortion }\end{array}$ & \\
\hline \multirow{9}{*}{ Assam } & All & 80.4 & 88.6 & 94.8 & 2.6 & 16.3 & 81.2 & 410 \\
\hline & Public & 74.6 & 82.1 & 90.5 & 4.6 & 19.4 & 75.9 & 226 \\
\hline & Hospitals & 85.7 & 94.4 & 91.8 & 0.0 & 10.2 & 89.8 & 67 \\
\hline & $\mathrm{CHCs}$ & 64.3 & 92.9 & 92.9 & 0.0 & 21.4 & 78.6 & 75 \\
\hline & PHCs & 75.0 & 62.5 & 87.5 & 12.5 & 25.0 & 62.5 & 84 \\
\hline & Private & 87.6 & 96.7 & 100.0 & 0.0 & 12.4 & 87.6 & 184 \\
\hline & Hospitals & 100.0 & 93.7 & 100.0 & 0.0 & 0.0 & 100.0 & 51 \\
\hline & $\begin{array}{l}\text { Nursing and } \\
\text { maternity homes }\end{array}$ & 79.7 & 97.4 & 100.0 & 0.0 & 20.3 & 79.7 & 112 \\
\hline & Clinics & 100 & 100 & 100 & 0 & 0 & 100 & 20 \\
\hline \multirow{9}{*}{ Bihar } & All & 86.2 & 72.0 & 85.1 & 11.0 & 13.7 & 75.3 & 1868 \\
\hline & Public & 68.8 & 53.0 & 76.9 & 18.0 & 30.5 & 51.5 & 227 \\
\hline & Hospitals & 57.7 & 81.5 & 92.8 & 1.6 & 40.7 & 57.7 & 104 \\
\hline & $\mathrm{CHCs}$ & 71.6 & 28.4 & 71.6 & 11.4 & 28.4 & 60.2 & 33 \\
\hline & PHCs & 80.6 & 29.1 & 60.6 & 39.4 & 19.4 & 41.2 & 90 \\
\hline & Private & 88.6 & 74.7 & 86.3 & 10.0 & 11.4 & 78.5 & 1641 \\
\hline & Hospitals & 100.0 & 66.9 & 83.0 & 17.0 & 0.0 & 83.0 & 132 \\
\hline & $\begin{array}{l}\text { Nursing and } \\
\text { maternity homes }\end{array}$ & 86.6 & 84.5 & 97.9 & 0.0 & 13.4 & 86.6 & 1047 \\
\hline & Clinics & 89.8 & 54.7 & 60.9 & 30.8 & 10.2 & 59.0 & 462 \\
\hline
\end{tabular}

Continued. 


\begin{tabular}{|c|c|c|c|c|c|c|c|c|}
\hline \multirow{2}{*}{ Facilities } & & \multicolumn{3}{|c|}{$\%$ of facilities offering each method } & \multicolumn{3}{|c|}{$\begin{array}{l}\text { \% distribution of facilities by method } \\
\text { category }\end{array}$} & \multirow[b]{2}{*}{$\mathbf{N}$} \\
\hline & & $\begin{array}{l}\text { MMA } \\
\text { (combi } \\
\text { packs) }\end{array}$ & $\begin{array}{l}\text { Vacuum } \\
\text { aspiration }\end{array}$ & $\begin{array}{l}\text { D and } \\
\text { C/D and } \\
E\end{array}$ & $\begin{array}{l}\text { Only } \\
\text { medical } \\
\text { abortion }\end{array}$ & $\begin{array}{l}\text { Only } \\
\text { surgical } \\
\text { abortion }\end{array}$ & $\begin{array}{l}\text { Both medical } \\
\text { and surgical } \\
\text { abortion }\end{array}$ & \\
\hline \multirow{10}{*}{ Gujarat } & All & 87.9 & 68.5 & 82.6 & 12.2 & 11.9 & 75.9 & 1392 \\
\hline & Public & 82.6 & 66.9 & 56.8 & 25.4 & 15.9 & 58.7 & 257 \\
\hline & Hospitals & 78.8 & 92.9 & 100.0 & 0.0 & 15.6 & 84.4 & 72 \\
\hline & CHCs & 83.6 & 61.7 & 61.7 & 38.3 & 16.4 & 45.3 & 80 \\
\hline & PHCs & 83.3 & 50.0 & 18.3 & 35.0 & 16.7 & 48.3 & 98 \\
\hline & Urban Public & 100.0 & 100.0 & 100.0 & 0.0 & 0.0 & 100.0 & 6 \\
\hline & Private & 89.1 & 68.8 & 88.5 & 9.2 & 10.9 & 79.8 & 1135 \\
\hline & Hospitals & 82.3 & 63.0 & 92.2 & 7.8 & 17.7 & 74.5 & 205 \\
\hline & $\begin{array}{l}\text { Nursing and } \\
\text { maternity homes }\end{array}$ & 90.2 & 70.9 & 89.1 & 8.0 & 9.8 & 82.2 & 895 \\
\hline & Clinics & 100.0 & 50.6 & 50.6 & 49.4 & 0.0 & 50.6 & 36 \\
\hline \multirow{10}{*}{$\begin{array}{l}\text { Madhya } \\
\text { Pradesh }\end{array}$} & All & 95.0 & 81.2 & 81.8 & 10.0 & 5.0 & 85.0 & 2941 \\
\hline & Public & 97.2 & 80.0 & 69.7 & 11.3 & 2.8 & 85.8 & 670 \\
\hline & Hospitals & 97.9 & 79.5 & 93.7 & 4.0 & 2.1 & 93.9 & 255 \\
\hline & CHCs & 94.0 & 85.7 & 62.7 & 7.1 & 6.0 & 86.9 & 231 \\
\hline & PHCs & 100.0 & 67.2 & 32.6 & 32.8 & 0.0 & 67.2 & 150 \\
\hline & Urban Public & 100.0 & 100.0 & 100.0 & 0.0 & 0.0 & 100.0 & 34 \\
\hline & Private & 94.4 & 81.6 & 85.4 & 9.7 & 5.6 & 84.7 & 2271 \\
\hline & Hospitals & 94.5 & 93.7 & 92.3 & 1.0 & 5.5 & 93.4 & 822 \\
\hline & $\begin{array}{l}\text { Nursing and } \\
\text { maternity homes }\end{array}$ & 93.4 & 81.8 & 91.8 & 5.6 & 6.6 & 87.8 & 1235 \\
\hline & Clinics & 100.0 & 33.4 & 21.0 & 66.6 & 0.0 & 33.4 & 213 \\
\hline \multirow{10}{*}{$\begin{array}{l}\text { Tamil } \\
\text { Nadu }\end{array}$} & All & 89.4 & 49.4 & 76.9 & 14.9 & 6.3 & 78.9 & 2883 \\
\hline & Public & 74.8 & 84.3 & 67.3 & 4.9 & 17.1 & 78.1 & 374 \\
\hline & Hospitals & 82.6 & 78.1 & 81.3 & 5.2 & 4.9 & 89.9 & 208 \\
\hline & CHCs & 61.9 & 100.0 & 57.1 & 0.0 & 33.3 & 66.7 & 94 \\
\hline & PHCs & 66.5 & 85.1 & 24.5 & 14.9 & 33.5 & 51.6 & 50 \\
\hline & Urban Public & 74.7 & 74.7 & 75.1 & 0.0 & 25.3 & 74.7 & 22 \\
\hline & Private & 91.6 & 44.2 & 78.3 & 16.3 & 4.7 & 79.0 & 2510 \\
\hline & Hospitals & 93.7 & 50.5 & 89.5 & 6.4 & 3.4 & 90.2 & 954 \\
\hline & $\begin{array}{l}\text { Nursing and } \\
\text { maternity homes }\end{array}$ & 89.3 & 44.5 & 78.5 & 15.4 & 5.9 & 78.7 & 1342 \\
\hline & Clinics & 97.3 & 14.2 & 26.8 & 67.0 & 2.7 & 30.3 & 213 \\
\hline \multirow{10}{*}{$\begin{array}{l}\text { Uttar } \\
\text { Pradesh }\end{array}$} & All & 88.7 & 47.1 & 70.9 & 27.2 & 10.1 & 62.7 & 2575 \\
\hline & Public & 89.0 & 41.1 & 65.5 & 33.4 & 9.5 & 57.1 & 551 \\
\hline & Hospitals & 91.2 & 63.0 & 81.3 & 18.7 & 6.5 & 74.8 & 141 \\
\hline & $\mathrm{CHCs}$ & 78.8 & 45.1 & 81.4 & 18.6 & 18.6 & 62.9 & 202 \\
\hline & PHCs & 96.7 & 16.5 & 38.0 & 58.4 & 3.3 & 38.3 & 162 \\
\hline & Urban Public & 100.0 & 44.0 & 44.0 & 56.0 & 0.0 & 44.0 & 45 \\
\hline & Private & 88.6 & 48.7 & 72.3 & 25.5 & 10.3 & 64.2 & 2025 \\
\hline & Hospitals & 87.2 & 53.9 & 95.3 & 4.7 & 12.8 & 82.5 & 329 \\
\hline & $\begin{array}{l}\text { Nursing and } \\
\text { maternity homes }\end{array}$ & 89.4 & 61.3 & 77.9 & 20.4 & 10.6 & 69.0 & 1167 \\
\hline & Clinics & 87.6 & 17.3 & 45.7 & 49.9 & 8.1 & 42.0 & 528 \\
\hline
\end{tabular}

All health facilities providing induced abortion services do not offer both medical and surgical abortions: $37 \%$ in Uttar Pradesh, 25\% in Bihar, 24\% in Gujarat, 21\% in Tamil Nadu, $19 \%$ in Assam, and $15 \%$ in Madhya Pradesh (Table 2). A lower percentage of public facilities than those private facilities in Assam, Bihar, Gujarat, and Uttar Pradesh provide both medical and surgical abortion. Even all the public hospitals providing induced abortion in the above states were found not to offer both medical and surgical abortion. The percentage of PHCs providing both medical and surgical abortions varies from $38 \%$ in Uttar Pradesh to $67 \%$ in Madhya Pradesh. Eighty-four percent of facilities in Assam commonly do not use medical abortion (MA/MMA) for the gestations of less than eight 
completed weeks (Table 3). The corresponding figures are 44\% in Bihar, $41 \%$ in Gujarat, 34\% in Uttar Pradesh, $29 \%$ in Tamil Nadu, and 19\% in Madhya Pradesh. About half of the facilities in Assam commonly use electric vacuum aspiration (EVA) $(78 \%$ of private facilities and $29 \%$ of public facilities) for gestations of less than eight completed weeks. The use of dilatation and curettage (D and C) for less than eight weeks of pregnancy varies from $5 \%$ in Madhya Pradesh to $23 \%$ in Uttar Pradesh.

Table 3: Among facilities providing abortion care, percent distribution of facilities providing different methods of abortion to less than 8 weeks gestation by ownership, 2015.

\begin{tabular}{|c|c|c|c|c|c|c|c|c|}
\hline States & & $\begin{array}{l}\text { Medical } \\
\text { abortion }\end{array}$ & $\begin{array}{l}\text { Manual } \\
\text { vacuum } \\
\text { aspiration }\end{array}$ & $\begin{array}{l}\text { Electric } \\
\text { vacuum } \\
\text { aspiration }\end{array}$ & $\begin{array}{l}\text { Dilatation } \\
\text { and } \\
\text { curettage }\end{array}$ & $\begin{array}{l}\text { Dilation } \\
\text { and } \\
\text { evacuation }\end{array}$ & Others & $\mathbf{N}$ \\
\hline \multirow{3}{*}{ Assam } & All & 16.3 & 17.3 & 50.7 & 7.8 & 7.1 & 0.7 & 410 \\
\hline & Public & 18.5 & 30.0 & 28.6 & 12.8 & 10.1 & 0.0 & 226 \\
\hline & Private & 13.7 & 1.6 & 78.1 & 1.6 & 3.3 & 1.6 & 184 \\
\hline \multirow{3}{*}{ Bihar } & All & 56.3 & 20.7 & 3.5 & 17.4 & 1.9 & 0.1 & 1864 \\
\hline & Public & 48.8 & 23.2 & 5.1 & 21.7 & 0.4 & 0.8 & 254 \\
\hline & Private & 57.5 & 20.3 & 3.3 & 16.7 & 2.2 & 0.0 & 1610 \\
\hline \multirow{3}{*}{ Gujarat } & All & 59.4 & 3.4 & 12.6 & 15.9 & 8.3 & 0.4 & 1392 \\
\hline & Public & 67.7 & 4.7 & 9.7 & 12.5 & 3.1 & 2.3 & 257 \\
\hline & Private & 57.5 & 3.2 & 13.2 & 16.6 & 9.5 & 0.0 & 1135 \\
\hline \multirow{3}{*}{ Madhya Pradesh } & All & 80.7 & 12.7 & 1.7 & 4.8 & 0.0 & 0.1 & 2942 \\
\hline & Public & 81.8 & 11.2 & 4.9 & 1.5 & 0.0 & 0.6 & 671 \\
\hline & Private & 80.3 & 13.1 & 0.7 & 5.8 & 0.0 & 0.0 & 2271 \\
\hline \multirow{3}{*}{ Tamil Nadu } & All & 71.3 & 12.3 & 1.6 & 13.4 & 0.6 & 0.9 & 2884 \\
\hline & Public & 45.6 & 43.5 & 1.6 & 6.9 & 0.0 & 2.4 & 374 \\
\hline & Private & 75.1 & 7.6 & 1.6 & 14.3 & 0.6 & 0.6 & 2510 \\
\hline \multirow{3}{*}{ Uttar Pradesh } & All & 66.3 & 5.1 & 4.6 & 22.6 & 0.8 & 0.7 & 2576 \\
\hline & Public & 64.7 & 8.7 & 5.6 & 15.5 & 2.4 & 3.1 & 551 \\
\hline & Private & 66.8 & 4.1 & 4.3 & 24.5 & 0.3 & 0.0 & 2025 \\
\hline
\end{tabular}

\section{Husband's and family member's consent for abortion procedures}

Women's consent for induced abortion is required before performing an abortion and is usually followed by the majority of the facilities across the state (Table 4). Nevertheless, $18 \%$ of the facilities in Bihar, $16 \%$ in Tamil Nadu, and $10 \%$ in Uttar Pradesh usually do not take women's consent before performing an abortion. Legally, the approval of the husband is not required. However, a sizable percentage of the facilities commonly take the permission of the husband $(65 \%$ in Madhya Pradesh to $92 \%$ in Tamil Nadu). About a quarter of Bihar, Tamil Nadu, and Uttar Pradesh facilities are also found to seek in-law's consent commonly. In Bihar, $44 \%$ of the facilities routinely take the parent's permission in case of unmarried women before offering induced abortion.

\section{Inadequate information/advice on procedure and follow- up care}

In Assam, Bihar, Madhya Pradesh, and Uttar Pradesh, less than half of the health facilities providing induced abortion inform women about the abortion procedure (Table 4). The corresponding figures are 60\% in Gujarat and 63\% in Tamil Nadu. A higher percentage of private facilities inform women about the abortion procedure in Bihar,
Gujarat, and Tamil Nadu. Advice on pain management/bleeding and infection prevention is very low, ranging from 2\% in Bihar to $17 \%$ in Madhya Pradesh. Advice on follow-up visits varies between $31 \%$ in Tamil Nadu to $77 \%$ in Assam.

\section{Turning away abortion seekers}

Eighty-seven percent of health facilities offering induced abortion in Bihar have turned away one or more women seeking an abortion last year, followed by $81 \%$ in Uttar Pradesh (Table 5). The corresponding figures are $69 \%$ in Assam, 56\% in Gujarat, 54\% in Madhya Pradesh, and 51\% in Tamil Nadu. Across states, a higher percentage of private facilities have turned away women except for Uttar Pradesh. There is no uniform pattern emerging in the association between turning away and the location of the facility. Abortion seekers being young/unmarried/have no child has been cited as the reason for turning away by a sizable percentage of facilities across states $(54 \%$ in Assam, 44\% in Uttar Pradesh, 37\% in Gujarat, 29\% in Bihar, 26\% in Madhya Pradesh and 22\% in Tamil Nadu).

A quarter of the facilities in Bihar and one-fifth of the facilities in Uttar Pradesh have cited no consent of the husband/family as the reason for the refusal to provide induced abortion. Non-availability of a provider or 
MA/MMA drugs has further been cited as a reason for turning away abortion seekers: $79 \%$ in Bihar, $77 \%$ in Uttar
Pradesh, 72\% in Madhya Pradesh, 67\% in Tamil Nadu, $57 \%$ in Assam, and Gujarat.

Table 4: Among facilities providing induced abortion services, proportion and percentage distribution of consent usually taken before offering abortion, and advice given while offering abortion, by ownership and location of facility, 2015.

\begin{tabular}{|c|c|c|c|c|c|c|c|c|c|}
\hline \multirow{2}{*}{ States } & & \multicolumn{4}{|c|}{$\%$ reporting consent } & \multicolumn{3}{|c|}{$\%$ reporting type of advice } & \multirow[b]{2}{*}{$\mathbf{N}$} \\
\hline & & $\begin{array}{l}\text { Women's } \\
\text { consent }\end{array}$ & $\begin{array}{l}\text { Husband's } \\
\text { consent }\end{array}$ & $\begin{array}{l}\text { In-law's } \\
\text { consent }\end{array}$ & $\begin{array}{l}\text { Parent's } \\
\text { consent }\end{array}$ & $\begin{array}{l}\text { What the } \\
\text { procedure } \\
\text { does }\end{array}$ & $\begin{array}{l}\text { Pain management/ } \\
\text { information on bleeding/ } \\
\text { infection prevention }\end{array}$ & $\begin{array}{l}\text { Follow } \\
\text { up visit }\end{array}$ & \\
\hline \multirow{5}{*}{ Assam } & All & 93.9 & 85.6 & 0.0 & 10.5 & 49.4 & 7.6 & 76.8 & 410 \\
\hline & Public & 90.3 & 76.5 & 0.0 & 7.5 & 44.7 & 4.9 & 80.5 & 226 \\
\hline & Private & 98.4 & 96.7 & 0.0 & 14.1 & 55.2 & 10.9 & 72.3 & 184 \\
\hline & Rural & 87.5 & 79.5 & 0.0 & 1.7 & 36.9 & 5.7 & 86.4 & 176 \\
\hline & Urban & 98.7 & 89.7 & 0.0 & 17.1 & 58.7 & 9.0 & 69.7 & 234 \\
\hline \multirow{5}{*}{ Bihar } & All & 82.3 & 76.9 & 26.3 & 44.0 & 47.9 & 1.6 & 62.7 & 1864 \\
\hline & Public & 64.8 & 69.2 & 18.5 & 45.8 & 65.6 & 7.1 & 53.5 & 254 \\
\hline & Private & 85.1 & 78.2 & 27.5 & 43.7 & 45.1 & 0.7 & 64.1 & 1610 \\
\hline & Rural & 70.7 & 72.5 & 36.4 & 64.2 & 67.7 & 3.4 & 58.0 & 324 \\
\hline & Urban & 84.9 & 77.9 & 24.2 & 39.7 & 43.7 & 1.2 & 63.6 & 1540 \\
\hline \multirow{5}{*}{ Gujarat } & All & 98.3 & 71.0 & 12.6 & 8.4 & 59.6 & 2.7 & 61.1 & 1392 \\
\hline & Public & 98.4 & 69.9 & 11.7 & 14.5 & 78.2 & 2.7 & 64.8 & 257 \\
\hline & Private & 98.2 & 71.2 & 12.9 & 7.0 & 55.3 & 2.7 & 60.3 & 1135 \\
\hline & Rural & 98.0 & 62.4 & 8.8 & 4.9 & 67.6 & 0.0 & 62.0 & 204 \\
\hline & Urban & 98.3 & 72.4 & 13.3 & 9.1 & 58.2 & 3.2 & 60.9 & 1188 \\
\hline \multirow{5}{*}{$\begin{array}{l}\text { Madhya } \\
\text { Pradesh }\end{array}$} & All & 92.0 & 64.7 & 11.0 & 6.0 & 44.1 & 16.7 & 54.2 & 2942 \\
\hline & Public & 91.5 & 57.8 & 8.1 & 11.0 & 45.0 & 16.9 & 61.6 & 671 \\
\hline & Private & 93.7 & 66.7 & 11.9 & 4.5 & 43.8 & 16.6 & 52.0 & 2271 \\
\hline & Rural & 98.1 & 56.2 & 5.6 & 1.2 & 36.6 & 16.5 & 32.7 & 322 \\
\hline & Urban & 91.3 & 65.7 & 11.7 & 6.6 & 45.0 & 16.6 & 56.9 & 2620 \\
\hline \multirow{5}{*}{$\begin{array}{l}\text { Tamil } \\
\text { Nadu }\end{array}$} & All & 84.1 & 91.5 & 23.3 & 8.1 & 63.3 & 5.7 & 30.9 & 2884 \\
\hline & Public & 83.4 & 87.7 & 18.7 & 10.7 & 67.4 & 5.6 & 22.5 & 374 \\
\hline & Private & 84.2 & 92.1 & 24.0 & 7.8 & 62.7 & 5.7 & 32.2 & 2510 \\
\hline & Rural & 88.4 & 82.3 & 3.4 & 7.5 & 67.3 & 6.8 & 26.7 & 147 \\
\hline & Urban & 83.8 & 92.0 & 24.3 & 8.2 & 63.1 & 5.7 & 31.1 & 2737 \\
\hline \multirow{5}{*}{$\begin{array}{l}\text { Uttar } \\
\text { Pradesh }\end{array}$} & All & 89.6 & 82.8 & 23.4 & 13.2 & 45.8 & 16.0 & 63.2 & 2576 \\
\hline & Public & 94.0 & 83.5 & 26.7 & 12.3 & 39.3 & 10.7 & 69.1 & 551 \\
\hline & Private & 88.4 & 82.6 & 22.5 & 13.4 & 47.6 & 17.4 & 61.6 & 2025 \\
\hline & Rural & 90.2 & 78.4 & 22.5 & 20.1 & 34.1 & 12.5 & 77.9 & 1033 \\
\hline & Urban & 89.2 & 85.7 & 24.0 & 8.5 & 53.6 & 18.3 & 53.3 & 1542 \\
\hline
\end{tabular}

Table 5: Among facilities providing induced abortion services, proportion and percentage distribution of facilities turning away abortion seekers and reasons, by ownership and location of facility, 2015.

\begin{tabular}{|c|c|c|c|c|c|c|}
\hline \multirow[b]{2}{*}{ States } & & \multicolumn{3}{|l|}{ Reasons } & \multirow{2}{*}{$\begin{array}{l}\text { Turned away } \\
\text { any abortion } \\
\text { seeker in last } \\
\text { year }\end{array}$} & \multirow[b]{2}{*}{$\mathbf{N}$} \\
\hline & & $\begin{array}{l}\text { Young/unmarried/no } \\
\text { children }\end{array}$ & $\begin{array}{l}\text { No consent } \\
\text { husband/family }\end{array}$ & $\begin{array}{l}\text { Provider/MA } \\
\text { not available }\end{array}$ & & \\
\hline \multirow{5}{*}{ Assam } & All & 54.3 & 7.8 & 56.5 & 68.9 & 410 \\
\hline & Public & 52.8 & 11.9 & 73.8 & 29.6 & 226 \\
\hline & Private & 56.1 & 2.4 & 34.1 & 32.8 & 184 \\
\hline & Rural & 67.2 & 9.5 & 71.5 & 78.3 & 176 \\
\hline & Urban & 41.8 & 6.2 & 42.1 & 62.0 & 234 \\
\hline \multirow{5}{*}{ Bihar } & All & 28.5 & 24.5 & 79.1 & 86.8 & 1864 \\
\hline & Public & 32.2 & 23.5 & 88.8 & 84.6 & 254 \\
\hline & Private & 27.9 & 30.8 & 77.6 & 87.2 & 1610 \\
\hline & Rural & 42.3 & 28.7 & 76.1 & 95.4 & 324 \\
\hline & Urban & 25.3 & 23.5 & 79.9 & 84.9 & 1540 \\
\hline Gujarat & All & 37.1 & 17.9 & 56.6 & 56.1 & 1392 \\
\hline
\end{tabular}

Continued. 


\begin{tabular}{|c|c|c|c|c|c|c|}
\hline \multirow[b]{2}{*}{ States } & & \multicolumn{3}{|l|}{ Reasons } & \multirow{2}{*}{$\begin{array}{l}\text { Turned away } \\
\text { any abortion } \\
\text { seeker in last } \\
\text { year }\end{array}$} & \multirow[b]{2}{*}{$\mathbf{N}$} \\
\hline & & $\begin{array}{l}\text { Young/unmarried/no } \\
\text { children }\end{array}$ & $\begin{array}{l}\text { No consent } \\
\text { husband/family }\end{array}$ & $\begin{array}{l}\text { Provider/MA } \\
\text { not available }\end{array}$ & & \\
\hline & Public & 22.2 & 6.7 & 59.6 & 35.0 & 257 \\
\hline & Private & 39.1 & 19.4 & 56.2 & 60.9 & 1135 \\
\hline & Rural & 13.2 & 5.5 & 44.0 & 44.6 & 204 \\
\hline & Urban & 40.2 & 19.4 & 58.3 & 58.1 & 1188 \\
\hline \multirow{5}{*}{ Madhya Pradesh } & All & 26.1 & 12.6 & 71.5 & 54.3 & 2942 \\
\hline & Public & 17.6 & 15.5 & 74.6 & 36.4 & 671 \\
\hline & Private & 27.7 & 12.0 & 71.0 & 59.6 & 2271 \\
\hline & Rural & 13.7 & 0.5 & 74.7 & 59.2 & 322 \\
\hline & Urban & 27.8 & 14.2 & 71.1 & 53.7 & 2620 \\
\hline \multirow{5}{*}{ Tamil Nadu } & All & 22.4 & 9.2 & 66.5 & 51.4 & 2884 \\
\hline & Public & 12.6 & 1.1 & 64.8 & 46.9 & 374 \\
\hline & Private & 23.7 & 10.3 & 66.7 & 52.1 & 2510 \\
\hline & Rural & 10.0 & 24.6 & 82.9 & 47.6 & 147 \\
\hline & Urban & 23.1 & 8.5 & 65.7 & 51.6 & 2737 \\
\hline \multirow{5}{*}{ Uttar Pradesh } & All & 44.1 & 21.2 & 77.2 & 81.0 & 2576 \\
\hline & Public & 48.5 & 17.4 & 86.9 & 88.9 & 551 \\
\hline & Private & 42.8 & 22.4 & 74.2 & 78.8 & 2025 \\
\hline & Rural & 54.9 & 19.5 & 79.9 & 77.4 & 1033 \\
\hline & Urban & 37.4 & 22.3 & 75.6 & 83.3 & 1542 \\
\hline
\end{tabular}

Table 6: Among facilities providing induced abortion services, proportion and percentage distribution of facilities putting contraception as a condition to offer abortion, type of women required to adopt contraception, and proportion of facilities encourage female sterilization acceptance by ownership and location of facility, 2015.

\begin{tabular}{|c|c|c|c|c|c|c|c|c|c|c|c|}
\hline & & & & Type of & omen re & lired to acc & contrac & & & & \\
\hline States & & $\begin{array}{l}\text { Encourage } \\
\text { women to } \\
\text { adopt } \\
\text { female } \\
\text { sterilization }\end{array}$ & $\mathbf{N}$ & 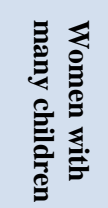 & 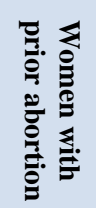 & 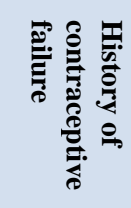 & 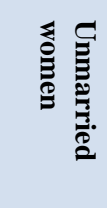 & 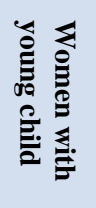 & 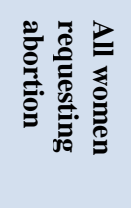 & $\begin{array}{l}\text { Put } \\
\text { contraception } \\
\text { as a condition } \\
\text { for abortion }\end{array}$ & $\mathbf{N}$ \\
\hline & All & 48.1 & 240 & 69.0 & 33.9 & 18.8 & 16.0 & 40.2 & 37.9 & 12.9 & 1864 \\
\hline & Public & 47.7 & 40 & 65.0 & 10.3 & 20.0 & 28.2 & 48.7 & 35.0 & 15.9 & 254 \\
\hline Bihar & Private & 50.0 & 200 & 69.8 & 38.5 & 18.5 & 13.6 & 38.5 & 38.5 & 12.4 & 1610 \\
\hline & Rural & 31.5 & 54 & 92.6 & 7.4 & 14.8 & 0.0 & 70.4 & 24.1 & 16.7 & 324 \\
\hline & Urban & 53.0 & 185 & 62.2 & 41.6 & 19.9 & 20.5 & 31.4 & 42.2 & 12.0 & 1540 \\
\hline & All & 26.9 & 274 & 28.1 & 11.7 & 20.4 & 20.0 & 49.6 & 19.3 & 20.3 & 1392 \\
\hline & Public & 51.2 & 80 & 28.8 & 23.8 & 15.0 & 32.5 & 43.8 & 48.8 & 31.2 & 257 \\
\hline Gujarat & Private & 16.9 & 194 & 27.8 & 6.7 & 22.7 & 14.9 & 52.1 & 7.2 & 17.7 & 1135 \\
\hline & Rural & 60.3 & 58 & 17.2 & 22.4 & 15.5 & 22.4 & 34.5 & 60.3 & 28.4 & 204 \\
\hline & Urban & 18.1 & 216 & 30.9 & 8.8 & 22.1 & 19.0 & 53.5 & 8.3 & 18.9 & 1188 \\
\hline & All & 30.5 & 761 & 42.8 & 48.1 & 23.8 & 16.3 & 53.0 & 20.9 & 25.9 & 2942 \\
\hline & Public & 43.9 & 157 & 38.9 & 56.7 & 34.4 & 4.5 & 66.2 & 12.7 & 23.4 & 671 \\
\hline Madhya & Private & 27.0 & 604 & 43.9 & 45.9 & 21.0 & 19.4 & 49.5 & 23.0 & 26.6 & 2271 \\
\hline & Rural & 75.6 & 41 & 5.0 & 95.0 & 0.0 & 0.0 & 87.8 & 0.0 & 12.7 & 322 \\
\hline & Urban & 28.0 & 721 & 44.9 & 45.5 & 25.2 & 17.2 & 50.9 & 22.1 & 27.5 & 2620 \\
\hline & All & 16.9 & 532 & 71.8 & 36.2 & 20.1 & 0.0 & 24.0 & 27.8 & 18.5 & 2884 \\
\hline & Public & 37.7 & 76 & 87.0 & 31.2 & 7.9 & - & 24.7 & 42.1 & 20.6 & 374 \\
\hline Tamil & Private & 13.4 & 456 & 69.2 & 37.1 & 22.1 & - & 23.9 & 25.4 & 18.2 & 2510 \\
\hline & Rural & 62.5 & 24 & 82.6 & 43.5 & 17.4 & - & 0.0 & 37.5 & 16.3 & 147 \\
\hline & Urban & 14.9 & 509 & 71.4 & 36.0 & 20.2 & - & 25.1 & 27.3 & 18.6 & 2737 \\
\hline & All & 46.7 & 210 & 46.9 & 66.7 & 7.7 & 1.0 & 55.0 & 52.9 & 8.3 & 2576 \\
\hline & Public & 43.5 & 85 & 50.0 & 68.2 & 8.2 & 2.4 & 43.5 & 45.9 & 15.9 & 551 \\
\hline $\begin{array}{l}\text { Uttar } \\
\text { Pradesh }\end{array}$ & Private & 48.8 & 125 & 44.8 & 65.6 & 7.3 & 0.0 & 62.9 & 57.6 & 6.3 & 2025 \\
\hline & Rural & 30.8 & 91 & 47.8 & 69.2 & 0.0 & 0.0 & 45.1 & 61.5 & 9.2 & 1033 \\
\hline & Urban & 58.5 & 117 & 46.2 & 65.3 & 13.7 & 1.7 & 62.7 & 46.2 & 7.8 & 1542 \\
\hline
\end{tabular}




\section{Contraception as a condition to offer induced abortion}

More than a quarter (26\%) of the health facilities in Madhya Pradesh put modern contraception as a condition to provide induced abortion (Table 6). Nearly one-fifth of the facilities in Gujarat and Tamil Nadu too ensure acceptance of contraception as a condition. A higher percentage of public facilities compared with private facilities in Bihar, Gujarat, Tamil Nadu, and Uttar Pradesh put contraception as a condition to offer induced abortion. In Bihar, facilities usually require women with many children (69\%) and women with a young child (40\%) to adopt contraception as a condition for abortion. Women with many children (72\%) followed by women with prior abortion $(36 \%)$ were required to accept contraception in Tamil Nadu. Women with young children were mainly needed to adopt contraception in Madhya Pradesh (53\%) and Gujarat (50\%). In Uttar Pradesh, $67 \%$ of the facilities report women with prior abortion, and $55 \%$ of facilities view women with young children are required to adopt a modern contraceptive method as a condition for receiving an abortion. About half of Bihar and Uttar Pradesh facilities encourage abortion seekers to take female sterilization as a contraceptive method. More than a quarter of abortion seekers are also encouraged to use female sterilization in Gujarat (27\%) and Madhya Pradesh $(31 \%)$.

\section{The restricted timing of PAC services provision}

Among the health facilities providing PAC services, more than three-fifths (64\%) in Madhya Pradesh do not offer PAC services all days throughout the week (Figure 2). The corresponding figures are 39\% in Uttar Pradesh, 27\% in Bihar, 24\% in Tamil Nadu, $18 \%$ in Assam, and $15 \%$ in Gujarat. Except for Madhya Pradesh, a higher percentage of public facilities in other states do not provide PAC services 24/7. Among the PHCs providing PAC services, $76 \%$ in Uttar Pradesh, 64\% in Madhya Pradesh, $48 \%$ in Bihar, $42 \%$ in Gujarat, $35 \%$ in Assam, and 24\% in Tamil Nadu do not provide $24 / 7$ services. As against urban facilities, a higher percentage of public facilities located in rural areas do not offer PAC services 24/7 in Assam, Bihar, Gujarat, and Uttar Pradesh (Not shown in the figure).

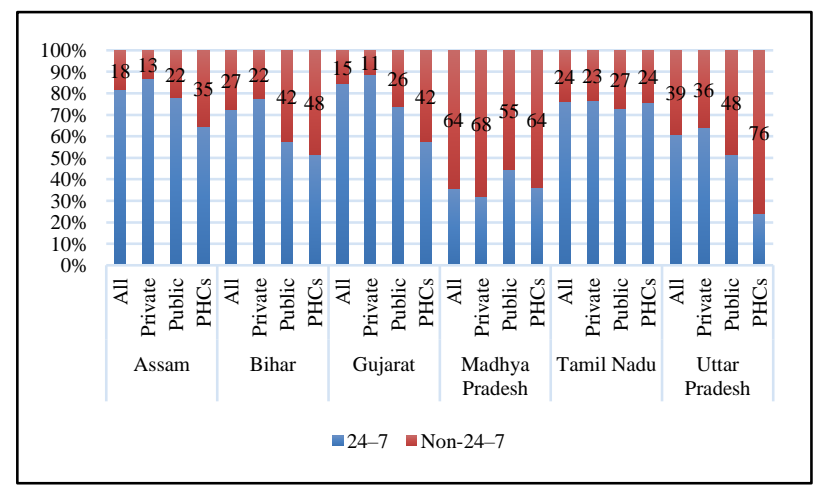

Figure 2: Among facilities providing PAC services, percent distribution according to time services are available, by ownership and type of facility, 2015.

\section{Non-medical reason for not providing induced abortion or PAC services}

Lack of trained staff and necessary equipment/supplies/space are cited as significant reasons for not providing MTP service, by a sizable percentage of health facilities providing only PAC services across states (Table not shown). Many facilities cited lack of facility certification as a reason for not giving MTP (47\% in Bihar, 40\% in Uttar Pradesh, 36\% in Gujarat, 28\% in Assam, 25\% in Madhya Pradesh, and 24\% in Tamil Nadu). Among the public facilities, $10 \%$ in Uttar Pradesh, 14\% in Madhya Pradesh, 20\% each in Assam and Gujarat, and 32\% in Bihar cited facility not certified for abortion provision. Religious or social reasons of the manager/doctor have also been stated as a reason by considerable percent of facilities across the states $(38 \%$ in Gujarat, $30 \%$ each in Uttar Pradesh and Bihar, 25\% in Tamil Nadu, 20\% in Assam, and $14 \%$ in Madhya Pradesh. Among the facilities providing only MTP services, lack of trained staff and necessary equipment/supplies/space are cited as significant reasons for not providing PAC service by a sizable percentage of facilities irrespective of state (Table not shown). Lack of trained staff as a reason varies from $55 \%$ in Tamil Nadu to $96 \%$ in Assam. Again, a higher percentage of public facilities cited this reason except in Bihar. All the facilities in Assam, compared with $32 \%$ of the facilities in Gujarat, cited a lack of necessary equipment/ supplies/space as the reason.

\section{DISCUSSION}

The study found access to safe abortion services remains inadequate, especially in rural areas, and the finding conforms to earlier studies. ${ }^{13,14}$ The insufficient facility capacity, misconceptions about facility certification, and religious or social beliefs of the manager or doctors are found to further curtail access to safe abortion services. A considerable size of PHCs does not provide both medical and surgical abortion services due to poor infrastructure and lack of trained staff, despite being the first level of public health facilities accessible to rural women. Government statistics confirm the shortage of PHCs besides poor infrastructure and shortfall of providers in the studied states. ${ }^{15}$ We found misconceptions among providers about which facilities are legally approved to offer abortion services preventing women from safe abortion seeking. Many past studies also report trained providers at public health facilities do not provide abortion services due to unawareness of the legality of abortion or a wrong impression that their facility is not legally approved to offer abortion services. ${ }^{16-18} \mathrm{~A}$ study in Maharashtra found that a sizable number of medical students consider abortion to be morally wrong, and their attitudes toward abortion were associated with religious beliefs besides many other variables. ${ }^{19}$

Medical abortion is easy to administer, reduces the chance of complications arising from other procedures, and is often preferred by women. ${ }^{20-22}$ The WHO recommends 
using MMA and manual vacuum aspiration (MVA) during the first trimester as the preferred abortion method. ${ }^{23}$ Nevertheless, many facilities found not using MA/MMA for gestations of less than eight completed weeks. Facilities continue to provide D and C, which is an obsolete method of surgical abortion and is suggested to be replaced by vacuum aspiration and or medical methods. $\mathrm{D}$ and $\mathrm{C}$ is considerably more painful for women and less safe than vacuum aspiration. ${ }^{24,25}$ Islam et al in their study on the management of early pregnancy failure in Bangladesh, found that MVA is safe, effective, cheaper, and complication is also less than D and C. ${ }^{26}$ Cochrane systematic review by Tuncalp et al also found MVA is faster, less painful, and associated with less blood loss and fewer complications than $\mathrm{D}$ and $\mathrm{C}$ in the management of incomplete miscarriage. ${ }^{27}$

Clinical practice handbook for safe abortion by WHO (2014) reemphasizes that within the framework of national laws, providers should promote and protect: women's and adolescents' health and their human rights; informed and voluntary decision-making; and autonomy in decisionmaking. ${ }^{28}$ Contrary to the existing law, many facilities across states commonly take the consent of the husband, in-laws, and parents in case of unmarried women before offering induced abortion. This may be due to incorrect knowledge about abortion laws or to protect themselves. ${ }^{29-}$ 31 Evidence reveals that providers were more likely to insist on spousal consent from adolescent women than from adult women. ${ }^{32}$ On the other hand, some facilities, mainly in Bihar, Tamil Nadu, and Uttar Pradesh, usually do not take women's consent before performing an abortion, violating women's basic medical ethics and rights.

Information about the abortion procedure is integral to informed consent, and providers are expected to inform the women about this before performing an abortion. We, however, found more than half of the facilities providing induced abortion in the states of Assam, Bihar, Madhya Pradesh, and Uttar Pradesh fail to do so. Ganatra and Hirve found that fewer providers in the state of Maharashtra had explained to women the abortion procedure they were about to undergo, and the situation worsened in the case of adolescent women. ${ }^{32}$ Another study in the state of Jharkhand too reveals women were rarely informed about alternative methods of abortion, possible complications and counseled about post-abortion contraception. ${ }^{33}$ Alike earlier studies, we also found minimal advice on pain management/bleeding and infection prevention in public and private health facilities. . $^{9,32,33}$

Health facilities have turned away women seeking an abortion- the majority citing non-medical reasons such as the women being too young, unmarried, and having no child. No consent of the husband or family was also cited as the reason for the refusal to offer induced abortion services, and the finding concurs with a past study. ${ }^{9}$ Turning away abortion seekers due to non-medical reasons compel women to avail of services from unsafe providers, often endangering their health. The cited causes are beyond the guidelines under the MTP act and are a violation of women's right to life. Voluntary counseling and the provision of contraceptive methods are essential elements of high-quality abortion care. We, however, found contraception as a condition to offer abortion is common except in Assam, and public facilities found to have stronger adherence. Insisting contraceptive acceptance to women with many or with young children and women with prior abortion disregard women's right to whether, when, and how many children to have. Evidence from small-scale studies on contraceptive use among abortion clients in India suggests many women do not receive the services they need, and among those who choose to adopt a method, many do not receive adequate counselin. ${ }^{32,34,35}$ Additionally, encouraging women to adopt female sterilization in an unconducive situation for informed choice might regret sterilization. Past studies on sterilization acceptance found many women having poststerilization health problems and often regret due to child loss experience and poor quality of services. ${ }^{36,37}$ A sizable percentage of health facilities, including a majority of the PHCs providing PAC services, do not offer the services 24/7. Post-abortion complications often require emergency treatment, and limited access to this emergency health requirement violates women's reproductive rights. Lack of privacy and confidentiality, cost of safe abortion, lack of knowledge about the location of the safe provider, and scarcity of certified providers were perceived to force women for unsafe abortion, and this finding conforms to earlier studies. ${ }^{16,33,38,39}$

The study's strengths are that it highlights reproductive rights violations in health facilities using a bigger sample size from a representative survey with a robust sampling method. The results are helpful for the formulation of new or strengthening existing policies and programs for safe abortion. New evidence on reproductive rights violation at health facilities is of immense use and opens avenues for immediate intervention at the facility level and further research on health providers' knowledge and attitude towards abortion care. However, the study analysed secondary data gathered through interviews of respondents deemed to be most knowledgeable about abortion services in the facility. There are possibilities that the respondents may not be fully aware of the abortion care procedures in the facility, especially in bigger health facilities with many providers.

\section{CONCLUSION}

To conclude, we found inadequate access to safe abortion care services often due to limited facility infrastructure and a lack of safe providers. The socio-religious affiliation of the providers/managers and ignorance about facility certification for MTP further curtail the access and violates women's rights to safe abortion. More extensive use of the $\mathrm{D}$ and $\mathrm{C}$ method against the WHO guideline and nonprovision of MA for less than eight completed weeks of gestation questions the knowledge and attitude of the 
providers and is a violation of women's right to the benefit of scientific progress. Against the law, facilities continue to seek the consent of family members and are found to have turned away women on this ground, violating women's right to life. Lack of informed consent on abortion procedures and minimal advice on pain management/bleeding and infection prevention further questions the facilities' ongoing practice, which disregards women's right to information and education. Putting contraception as a condition to offer induced abortion, disregard women's right to whether and when to have children. Further, encouraging women to adopt female sterilization when they are not able to make an informed choice violates women's right to be free to choose and to use a method that is safe and acceptable to them. Results suggest the need to improve access to facility-based abortion services, especially in underserved rural areas, by ensuring that all public-sector facilities have adequate equipment and supplies, including MMA drugs and trained providers. Providing high-quality abortion care that adheres to international guidelines by training providers in current abortion techniques and best practices seems pertinent. Moreover, sensitization of health care providers about the importance of ethical issues and reproductive rights of women is required to ensure safe, legal, and accessible abortion care, which promotes health and justice for women.

Funding: No funding sources

Conflict of interest: None declared

Ethical approval: Not required

\section{REFERENCES}

1. United Nation. Reproductive Rights Are Human Rights. A Handbook for National Human Rights Institutions. United Nations Population Fund; 2014.

2. UNFPA. International Conference on Population and Development: Program of Action. United Nations Population Fund; 2004.

3. Ganatra B, Gerdts C, Rossier C, Johnson Jr BR, Tunçalp Ö, Assifi A, et al. Global, regional, and subregional classification of abortions by safety, 2010-14: estimates from a Bayesian hierarchical model. Lancet. 2017;390(10110):2372-81.

4. Sedgh G, Bearak J, Singh S, Bankole A, Popinchalk A, Ganatra B, et al. Abortion incidence between 1990 and 2014: global, regional, and subregional levels and trends. Lancet. 2016;388(10041):258-67.

5. Singh S, Shekhar C, Acharya R, Moore AM, Stillman M, Pradhan MR, et al. The incidence of abortion and unintended pregnancy in India, 2015. Lancet Glob Health. 2018;6(1):e111-20.

6. Hirve SS. Abortion law, policy and services in India: a critical review. Reprod Health Matters. 2004;12(24):114-21.

7. Stillman M, Frost JJ, Singh S, Moore AM, Kalyanwala S. Abortion in India: a literature review. New York: Guttmacher Institute; 2014:12-14.
8. Creanga AA, Roy P, Tsui AO. Characteristics of abortion service providers in two northern Indian states. Contraception. 2008;78(6):500-6.

9. Duggal R, Ramachandran V. The abortion assessment project- India: key findings and recommendations. Reprod Health Matters. 2004;12(24):122-9.

10. Grimes DA, Benson J, Singh S, Romero M, Ganatra B, Okonofua FE, et al. Unsafe abortion: the preventable pandemic. Lancet. 2006;368(9550):190819.

11. Banerjee $S$. Increasing access to safe abortion services in Uttarakhand: identifying medical termination of pregnancy training centers. New Delhi: IPAS India; 2007.

12. Kosgi S, Hegde VN, Rao S, Bhat US, Pai N. Women reproductive rights in India: prospective future. Online J Health Alli Sci. 2011;10(1):1-5.

13. Ipas. Improving access to comprehensive abortion care in India with focus on expanding the provider base. A policy brief. 2018.

14. Jejeebhoy SJ, Kalyanwala S, Mundle S, Tank J, Zavier AF, Kumar R, et al. Feasibility of expanding the medication abortion provider base in India to include ayurvedic physicians and nurses. Int Perspect Sex Reprod Health. 2012:133-42.

15. MoHFW, Rural Health Statistics. Ministry of Health and Family Welfare: New Delhi; 2016.

16. Jejeebhoy SJ, Zavier AJ, Acharya R, Kalyanwala S. Increasing access to safe abortion in rural Maharashtra: outcomes of a comprehensive abortion care model. Population council. 2011.

17. Navin D, Gulati S, Warvadekar J, Banerjee SK. Improving Comprehensive Abortion Care Services in Chhattisgarh through State Government- Ipas partnership- a facility baseline assessment, 2010. New Delhi, India: Ipas; 2011.

18. Patel L, Bennett TA, Halpern CT, Johnston HB, Suchindran CM. Support for provision of early medical abortion by mid-level providers in Bihar and Jharkhand, India. Reprod Health Matters. 2009; 17(33):70-9.

19. Sjöström S, Essén B, Sydén F, Gemzell-Danielsson K, Klingberg-Allvin M. Medical students' attitudes and perceptions on abortion: a cross-sectional survey among medical interns in Maharastra, India. Contraception. 2014;90(1):42-6.

20. Ganatra B, Kalyanwala S, Elul B, Coyaji K, Tewari S. Understanding women's experiences with medical abortion: in-depth interviews with women in two Indian clinics. Glob Public Health. 2010;5(4):335-47.

21. Shuchita M, Shveta K, Batya E, Suresh U. Simplifying medical abortion: home administration of misoprostol. J Obstet Gynecol India. 2008;58:410-6.

22. Sri BS, Ravindran TK. Medical abortion: understanding perspectives of rural and marginalized women from rural South India. Int J Gynaecol Obstet. 2012;118(1):S33-9.

23. World Health Organization, Department of Reproductive Health and Research. Safe abortion: technical and policy guidance for health systems 
evidence summaries and grade tables. 2nd edn. World Health Organization; 2012.

24. Grimes DA, Schultz KF, Cates Jr W, Tyler CW. The Joint Program for the Study of Abortion/CDC: a preliminary report. In: Abortion in the seventies: proceedings of the Western Regional Conference on Abortion. New York, NY: National Abortion Federation; 1977:41-54.

25. Cates W, Grimes DA, Schulz KF. Abortion surveillance at CDC: creating public health light out of political heat. Am J Prev Med. 2000;19(1):12-7.

26. Islam R, Biswas SP, Halder D, Fatima K. Safety and efficacy of manual vacuum aspiration compared to dilatation and curettage in the management of early pregnancy failure. Bangladesh Med $\mathrm{J}$ Khulna. 2016;49(1-2):18-22.

27. Tunçalp Ö, Gülmezoglu AM, Souza JP. Surgical procedures for evacuating incomplete miscarriage. Cochrane Database Syst Rev. 2010(9):Cd001993.

28. World Health Organization, Clinical practice handbook for safe abortion. World Health Organization; 2014.

29. Ganatra B. Abortion research in India: What we know, and what we need to know. Women's Reprod Health India. 2000:186-235.

30. Gupte M, Bandewar S, Pisal H. Women's perspectives on the quality of general and reproductive health care: Evidence from rural Maharashtra. Improving Quality Care in India's Family Welfare Programme. Population Council, New York; 1999:117-39.

31. Visaria L, Ramachandran V, Ganatra B, Kalyanwala S. Abortion in India: emerging issues from qualitative studies. Econom Polit Week. 2004:5044-52.
32. Ganatra B, Hirve S. Induced abortions among adolescent women in rural Maharashtra, India. Reprod Health Matters, 2002;10(19):76-85.

33. Barua A, Apte H. Quality of abortion care: perspectives of clients and providers in Jharkhand. Econom Polit Weekly. 2007:71-80.

34. Benson J, Andersen K, Brahmi D, Healy J, Mark A, Ajode $\mathrm{A}$, et al. What contraception do women use after abortion? An analysis of 319,385 cases from eight countries. Glob Public Health. 2018;13(1):3550.

35. Zavier A, Padmadas SS. Postabortion contraceptive use and method continuation in India. Int $\mathbf{J}$ Gynecol Obstet. 2012;118(1):65-70.

36. Pradhan MR, Ram U. Female sterilization and ethical issues: The Indian experience. Soc Change. 2009;39(3):365-87.

37. Ramanathan M, Mishra U, Correlates of female sterilization regret in the southern states of India. J Biosoc Sci. 2000;32(4):547-58.

38. Elul B, Barge S, Verma S, Kumar N, Bracken H, Sadhvani H. Unwanted pregnancy and induced abortion: data from men and women in Rajasthan, India. New Delhi: Population Council; 2004:29-30.

39. Banerjee SK, Andersen KL, Baird TL, Ganatra B, Batra S, Warvadekar J. Evaluation of a multi-pronged intervention to improve access to safe abortion care in two districts in Jharkhand. BMC Health Serv Res. 2014;14(1):1-2.

Cite this article as: Pradhan MR, Shekhar C, Alagarajan M, Sahoo H. Abortion care-seeking and reproductive rights violation in health facilities: evidence from six states of India. Int J Reprod Contracept Obstet Gynecol 2022;11:221-31. 